Original article

Radiologe 2021 - 61 (Suppl 1):S39-S48 https://doi.org/10.1007/s00117-020-00781-4 Accepted: 18 November 2020

Published online: 4 January 2021

(c) Springer Medizin Verlag GmbH, ein Teil von Springer Nature 2020

\section{Introduction}

During the past few decades, significant technical and methodological innovations have paved the way for highly precise and focused radiotherapy delivery. On the one hand, modern imaging technologies such as computed tomography (CT), magnetic resonance imaging (MRI), or positron emission tomography (PET) have become essential techniques for diagnostic evaluation and treatment planning in radiation therapy, allowing for accurate differentiation between cancerous and healthy tissue. Additionally, the integration of various imaging modalities directly into linear accelerators has enabled daily monitoring of patient positioning, tumor position, and alterations in patient anatomy.

Today, X-ray-based image guidance has become a widely used standard, as it is established in nearly all radiotherapy units, mostly by means of integrated cone-beam and megavoltage fan-beam CT (CBCT and MVCT). However, besides exposing the patient to additional radiation, CBCT and MVCT imaging is limited with respect to the achievable soft-tissue contrast, practically only allowing image guidance based on bony structures. On the other hand, MRI offers excellent soft-tissue contrast for

The authors C. Katharina Spindeldreier and Sebastian Klüter contributed equally to this article.

\author{
C. Katharina Spindeldreier ${ }^{1,2,3} \cdot$ Sebastian Klüter ${ }^{1,2,3} \cdot$ Philipp Hoegen $^{1,2,3} \cdot$ \\ Carolin Buchele $e^{1,2,3} \cdot$ Carolin Rippke $^{1,2,3} \cdot$ Eric Tonndorf-Martini ${ }^{1,2,3}$. \\ Jürgen Debus ${ }^{1,2,3,4,5}$ • Juliane Hörner-Rieber ${ }^{1,2,3,5}$ \\ 'Department of Radiation Oncology, Heidelberg University Hospital, Heidelberg, Germany \\ ${ }^{2}$ Heidelberg Institute of Radiation Oncology (HIRO), Heidelberg, Germany \\ ${ }^{3}$ National Center for Tumor diseases (NCT), Heidelberg, Germany \\ ${ }^{4}$ Heidelberg lon-Beam Therapy Center (HIT), Department of Radiation Oncology, Heidelberg University \\ Hospital, Heidelberg, Germany \\ ${ }^{5}$ Clinical Cooperation Unit Radiation Oncology, German Cancer Research Center (DKFZ), Heidelberg, \\ Germany
}

\title{
MR-guided radiotherapy of moving targets
}

the precise identification of target volume and immediate detection of interand intra-fractional changes of the tumor and adjacent organs at risk. Since the direct integration of MRI into medical linear accelerators has been challenging for many years, the first studies on MR-guided radiotherapy (MRgRT) focused on offline solutions [1-3]. Only recently have hybrid machines that combine MRI scanners with radiotherapy delivery systems become clinically available, enabling online MRgRT $[4,5]$. Two hybrid devices are currently in clinical use: The ViewRay (Mountain View, CA, USA) MRIdian Linac system combines a split-bore 0.35 -T MRI scanner with a radiation gantry including the linear accelerator (6 MV; [6]), while the Elekta (Stockholm, Sweden) Unity MR-Linac is composed of a 1.5-T MRI scanner and a ring-based gantry containing a 7-MV linear accelerator $[7,8]$.

The new hybrid MR-Linac systems not only offer superior anatomical 3D imaging for precise delineation of the tumor as well as immediate detection of interfractional changes, but also provide realtime information by cine MRI, allowing for constant monitoring of tumor volume and nearby critical structures during the entire treatment session [7,9]. Compared with conventional radiotherapy techniques, safety margins and hence the irradiated volume can be decreased, thereby reducing the risk of toxicity. In general, safety margins are applied in ra- diotherapy to ensure optimal dose coverage of the target volume besides uncertainties during planning, setup, or treatment [10]. MR-guided radiotherapy of smaller, clearly visible target volumes further has the potential of dose escalation in the tumor volume for increasing local control. Highly encouraging initial results have been published for several tumor entities including pancreatic carcinoma, early-stage low-risk breast cancer, prostate cancer as well hepatic and adrenal metastases [11-16].

Abdominal organs are subject to movement caused by breathing and positional drifts in the body of several centimeters during irradiation. For CTguided radiotherapy, clinically established strategies for motion compensation include breath-hold techniques or continuous irradiation in free breathing using an internal target volume (ITV). The ITV concept, which is most widely applied, accounts for tumor movement by incorporating the tumor position into the target volume in all breathing phases, assessed by a 4D CT [17]. Thus, with the ITV concept, the whole area of potential tumor position is irradiated.

By contrast, some MRgRT devices enable gated dose delivery. With MRguided gating, radiation is only delivered when the tracked tumor is in the right position in the cine MRI, and the treatment beam automatically turns off if the tumor moves outside a specified boundary [9]. The required safety margins and 


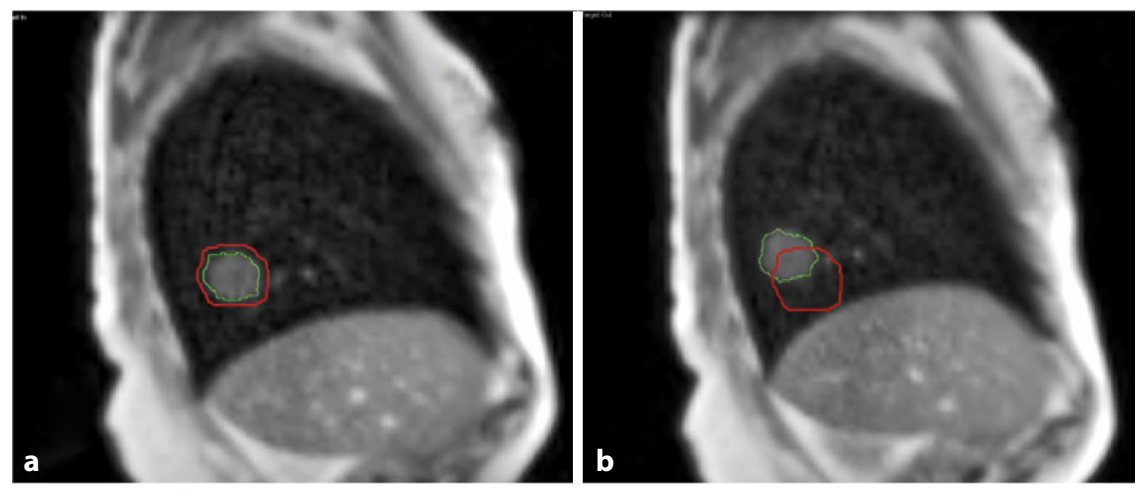

Fig. $1 \Delta$ Automated beam gating based on cine-magnetic resonance imaging in a sagittal plane through the tumor. Beam is on while the target (green) is within bounds (red; a) and automatically turned off when the target is outside the defined boundary (b)

hence treated volumes can thereby be reduced compared with the ITV concept (see • Fig. 1).

In this work, we present three clinical examples (lung metastasis, adrenal metastasis, and liver metastasis) for gated radiotherapy based on real-time cineMRI, as well as a comparison for those cases with retrospectively calculated ITV-based treatment plans.

\section{Material and methods}

\section{MR-Linac}

The MRIdian Linac that has been in clinical use in our institution since April 2018 provides a "true fast imaging with steady-state precession" (TRUFI) pulse sequence, a type of balanced steady-state free precession sequence (bSSFP), which can be used for volumetric imaging as well as cine imaging [6]. Moreover, navigator-based T1-weighted and T2-weighted images can be acquired in research mode. The clinical TRUFI sequence yields T2/T1-weighted contrast that highlights fat and water, enabling a straightforward differentiation of organs.

In MRgRT, volumetric imaging is used for treatment planning as well as patient positioning. Moreover, the treatment plan can be adapted online based on the daily patient anatomy while the patient lies on the treatment couch. The volumetric TRUFI can be acquired at the MRIdian Linac with an in-plane resolution of $1.5 \mathrm{~mm}$ and slice thicknesses of 1.5 or $3.0 \mathrm{~mm}$. During treatment delivery, sagittal 2D cine-MR images can be acquired at 4 frames/s with $3.5-\mathrm{mm}$ in-plane resolution and with 5-, 7-, or 10-mm slice thickness [6]. The cineMRI is used for real-time tumor tracking and automated beam gating. For tumor tracking, a key frame of the cineMRI is selected and registered to the volumetric image via deformable image registration. All subsequent cine images are registered to this key frame, which enables tracking of the target structure delineated in the volumetric image [9]. At Heidelberg University Hospital, gated MRgRT is performed with optional live video assistance. A video screen that can be observed by the patient via a mirror is installed at the end of the bore [18]. Patients can follow the position of the target volume in real time and breathe accordingly in order to steer their tumor to the desired position.

\section{Treatment planning}

The gross tumor volume (GTV) was delineated on the 3D MR image generated at the MR-Linac, and with the aid of contrast-enhanced CT-images as well as additional diagnostic MRI, if available. An appropriate clinical target volume margin (CTV) was added to account for microscopic infiltration. The resulting CTV was enlarged by $3 \mathrm{~mm}$ to form the planning target volume (PTV). In radiotherapy, the target volume, which comprises the macroscopic tumor (GTV) plus the microscopic tumor infiltration (CTV), is enlarged by a further margin to account for uncertainties during planning, setup, or treatment (PTV;
[10]). Step-and-shoot intensity-modulated radiotherapy (IMRT) treatment plans for the MR-Linac were generated using the ViewRay treatment planning system based on a Monte Carlo dose calculation algorithm considering the magnetic field. The dose calculation was carried out with a 3-mm dose grid and an uncertainty of $0.5 \%$.

For the ITV approach, comparative plans were calculated using RayStation (Version 8B 8.1.1.8, RaySearch, Stockholm, Sweden) with a collapsed cone dose calculation algorithm for an Elekta Versa HD Linac with 6 MV. Craniocaudal and anteroposterior ITV margins were estimated using the sagittal 2D cine images of the MR-Linac treatments [19], while a lateral margin of $2 \mathrm{~mm}$ was assumed in accordance with the literature [20, 21]. For the lung, liver, and adrenal patients, ITV margins of $1.1 \mathrm{~cm}, 1.7 \mathrm{~cm}$, and $1.75 \mathrm{~cm}$ in craniocaudal direction and $0.85 \mathrm{~cm}, 0.65 \mathrm{~cm}$, and $1.25 \mathrm{~cm}$ in anteroposterior direction, respectively, were determined from the real-time cine-MRimages recorded during therapy. The comparative plans were generated according to clinical standards used at Heidelberg University Hospital. For the adrenal gland and liver tumor, volumetric-modulated arc therapy (VMAT) plans were created, while a $3 \mathrm{D}$ conformal plan was calculated for the lung case. The PTV margin was set to $5 \mathrm{~mm}$ for all cases. MRLinac and ITV-based plans were generated using the same dose prescription and clinical goals for target coverage as well as organ-at-risk (OAR) dose constraints. Of note, in clinical routine treatment, these plans would not have been applied, as PTV coverage had to be reduced due to the proximity of critical radiosensitive OARs. In general, radiotherapy doses would instead have been adjusted and treatment would have been performed with reduced doses.

The treatment plans were compared based on PTV volume, target coverage, and dose-volume parameters of OARs. In order to compare the dose-volume parameters premised on the same calculation basis, the MR-Linac plans were imported into RayStation. 
Radiologe 2021 - 61 (Suppl 1):S39-S48 https://doi.org/10.1007/s00117-020-00781-4

(c) Springer Medizin Verlag GmbH, ein Teil von Springer Nature 2020

\section{K. Spindeldreier · S. Klüter · P. Hoegen · C. Buchele · C. Rippke · E. Tonndorf-Martini · J. Debus · J. Hörner-Rieber}

\section{MR-guided radiotherapy of moving targets}

\section{Abstract}

Introduction. Hybrid magnetic resonance (MR) linear accelerators (MR-Linacs) for radiotherapy allow for the visualization and tracking of moving target volumes during the entire treatment. This makes gated treatments possible, decreasing the irradiated volumes and thus sparing healthy tissue from unnecessary radiation dose. Conventionally, tumors that are subject to respiration motion are treated by irradiating the entire area of potential target presence (internal target volume, ITV). This study presents three patient cases (lung, adrenal gland, and liver tumors) treated with gated MR-guided radiotherapy and compares the treatment plans retrospectively with conventional ITV plans.
Materials and methods. The gross tumor volume was delineated on MR and computed tomography (CT) images of the patients, and MR-Linac treatment plans were generated using additional clinical and planning target volume margins. The motion of the gross tumor volume was evaluated on twodimensional cine-MRI images during the entire MR-Linac treatment. Based on the motion analysis, standard ITV-based plans were retrospectively created and compared by means of irradiated target volumes and dose-volume parameters.

Results. For the MR-Linac plans, the irradiated treatment volumes were reduced by an average of $62 \%$ across the three cases, and for one case the ITV-based target volume would have overlapped with a critical organ. Target volume coverage was much better and the lung and adrenal MR-Linac plans revealed superior sparing of the organs at risks thanks to gated treatments.

Conclusion. Dosimetrically beneficial treatment plans with promising clinical outcomes can be applied when using gated MR-guided radiotherapy. Future studies will reveal which patients will benefit most from this technique. To utilize the full potential of online adaptive, individualized MR-guided therapy, the close collaboration of radiooncology and radiology is needed.

\section{Keywords}

Magnetic resonance imaging · Organs at risk . Radiation dosage - Tumor burden · Radiooncology

\section{MR-gestützte Strahlentherapie beweglicher Zielobjekte}

\section{Zusammenfassung}

Hintergrund. Hybrid-Magnetresonanz(MR)Linearakzeleratoren (MR-Linacs) für die Strahlentherapie ermöglichen die Visualisierung und Verfolgung beweglicher Zielvolumina während der gesamten Behandlung. Das erlaubt atemgesteuerte Therapien, vermindert die bestrahlten Volumina und schont somit gesundes Gewebe vor unnötiger Strahlendosis. Konventionell werden Tumoren, die der Atembewegung ausgesetzt sind, durch Bestrahlung des gesamten Bereichs behandelt, in dem sich das Ziel befinden kann (,internal target volume", ITV). In der vorliegenden Studie werden 3 Fälle vorgestellt (Lungen-, Nebennieren- und Lebertumor), die mittels atemgesteuerter MRgeführter Strahlentherapie behandelt wurden, dabei werden die Behandlungsplanungen retrospektiv mit konventionellen ITV-

Planungen verglichen.

Material und Methoden. Das makroskopische Tumorvolumen wurde auf MRT- und
Computertomographie(CT)-Aufnahmen der Patienten abgegrenzt, und die jeweilige MRLinac-Behandlungsplanung wurde unter Berücksichtigung zusätzlicher klinischer und Planungszielvolumenmargen erstellt. Die Bewegung des makroskopischen Tumorvolumens wurde auf zweidimensionalen CINEMRT-Bildern während der gesamten MRLinac-Behandlung beurteilt. Auf Grundlage der Bewegungsanalyse wurden retrospektiv Standard-ITV-Planungen erstellt und anhand von bestrahlten Zielvolumina und DosisVolumen-Parametern verglichen.

Ergebnisse. Für die jeweilige MR-LinacPlanung wurde in den 3 Fällen das bestrahlte Behandlungsvolumen um durchschnittlich $62 \%$ reduziert, und in einem Fall hätte das ITV-basierte Zielvolumen sich mit einem kritischen Organ überschnitten. Die Zielvolumenabdeckung war viel besser und die MR-Linac-Planung für die Lunge und die Nebenniere wiesen eine deutlichere
Schonung der gefährdeten Organe durch die atemgesteuerte Behandlung auf.

Schlussfolgerung. Eine dosimetrisch vorteilhafte Behandlungsplanung mit vielversprechenden klinischen Ergebnissen kann eingesetzt werden, wenn die atemgesteuerte MR-geführte Strahlentherapie angewandt wird. Zukünftige Studien werden zeigen, welche Patienten den größten Vorteil durch diese Technik haben. Um das ganze Potenzial der online adaptiven, individualisierten MR-geführten Therapie zu nutzen, ist eine enge Zusammenarbeit zwischen der Radioonkologie und der Radiologie erforderlich.

\section{Schlüsselwörter}

Magnetresonanztomographie · Risikoorgane . Bestrahlungsdosierung - Tumorlast . Radioonkologie

\section{Case presentation and results}

\section{Patient cases}

To highlight the potential of MRgRT, we evaluated the treatments of three patients treated at the MRIdian Linac at the Department of Radiation Oncology of Heidelberg University Hospital, Germany. All patients have been included in a prospective registry (Heidelberg Medical Faculty Ethics Commission, study ID S-506/2018).

The first patient (female, age 82) had initially been diagnosed with a large-cell neuroendocrine carcinoma (pT4pN0cM0) of the lung, and had therefore undergone surgical resection of the right lower lobe as well as mediastinal lymphadenectomy 5 years before. A growing lung nodule of the left upper lobe was diagnosed in June 2019. The patient underwent positron-emission tomography (PET) for staging and to rule out 


\section{Original article}
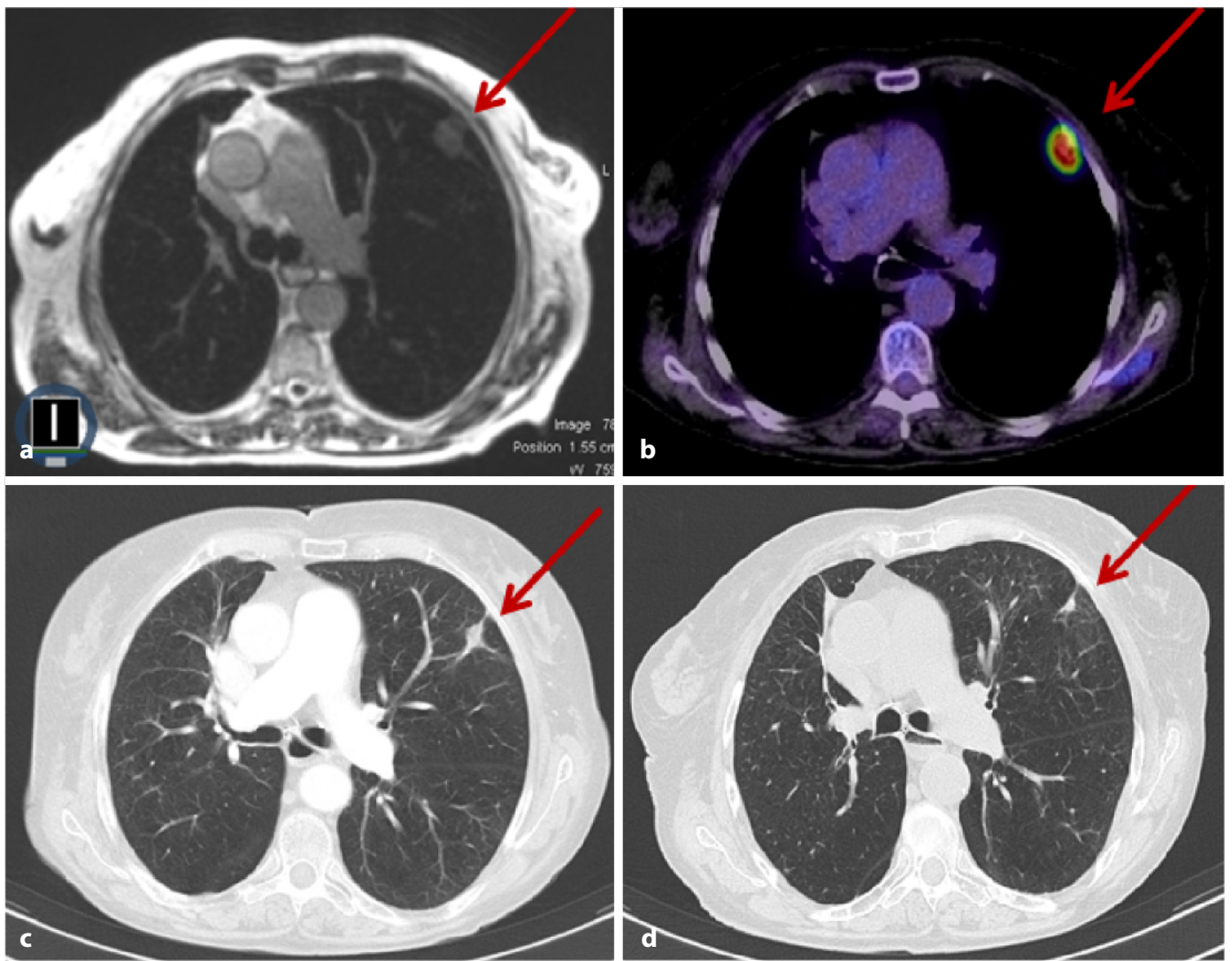

Fig. $2<$ Lung metastasis in the upper left lobe (red arrow): a TrueFiSP at the MR-Linac for treatment planning; $\mathbf{b}$ fluorodeoxyglucose positron emission tomography (FDG-PET) computed tomography (CT) for staging; c and d contrast-enhanced CT scan 3 months and 6 months after MR-guided radiotherapy of the lung metastasis
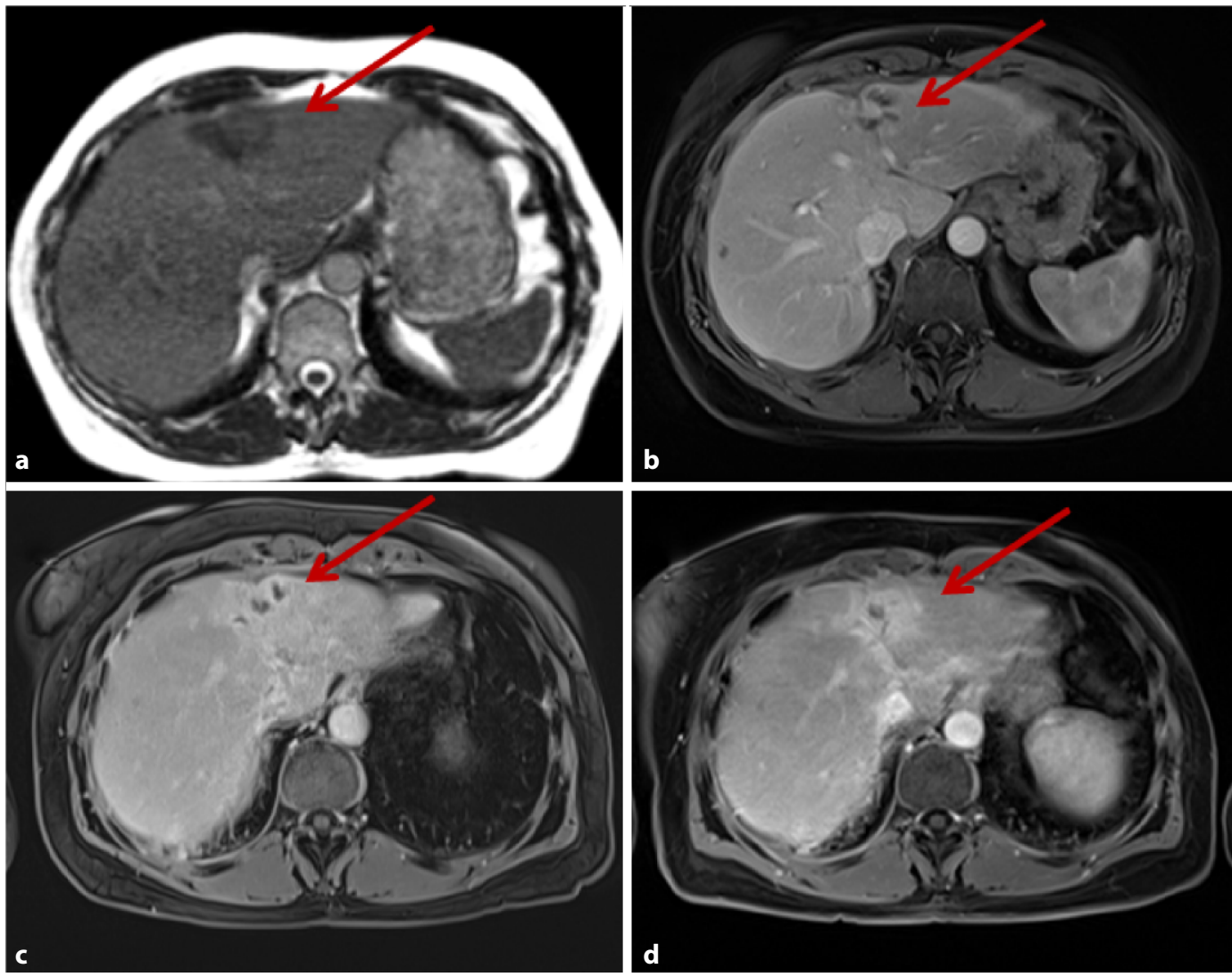

Fig. $3<$ Liver metastasis in segments II/IV (red arrow): a TrueFiSP at the MR-Linac for treatment planning; $b$ post-contrast T1-weighted image from clinical scanner for planning; $c$ and d postcontrast T1-weighted images from clinical scanner 3 months and 9 months after MR-guided radiotherapy of the liver metastasis 

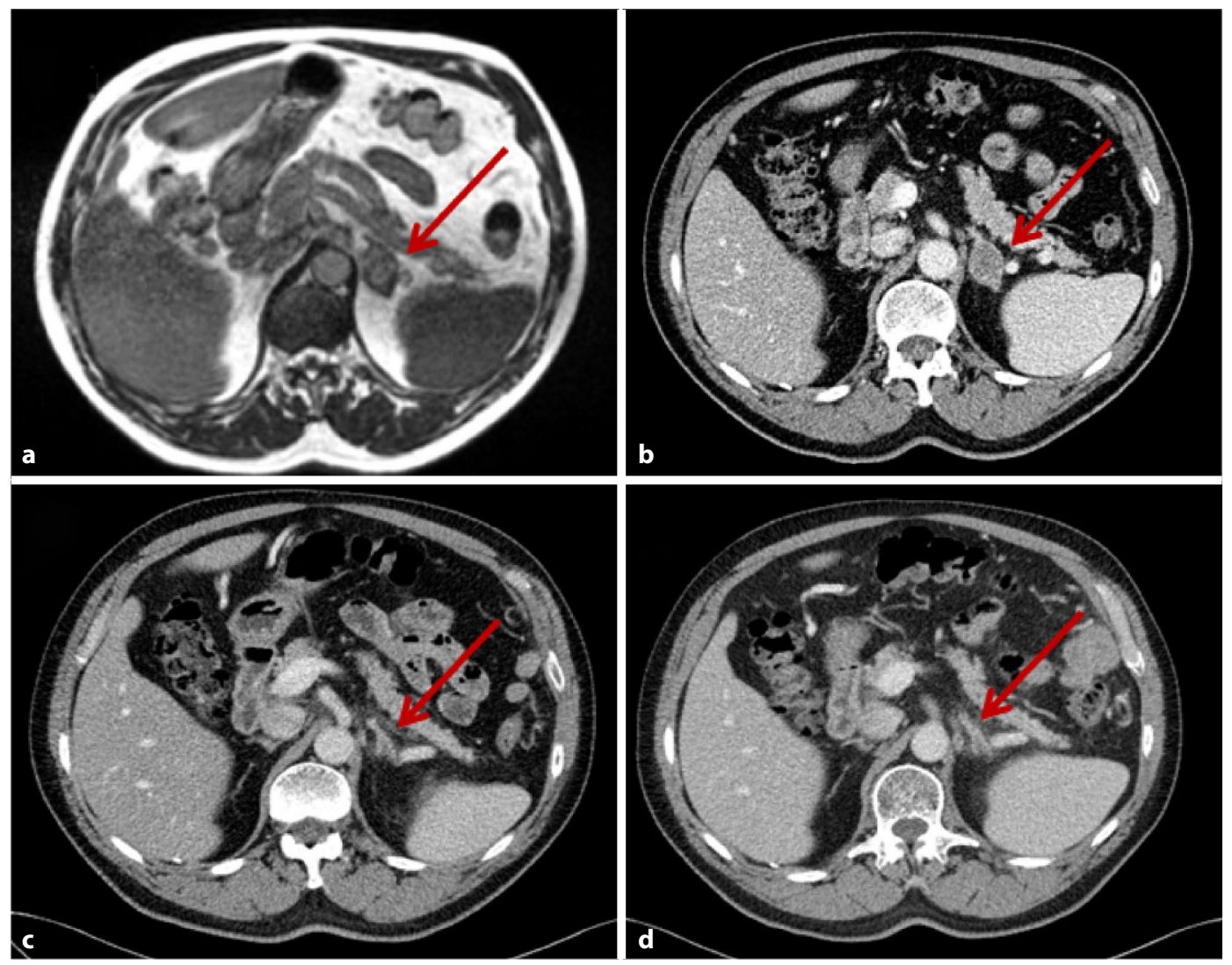

Fig. $4<$ Left adrenal metastasis (red arrow): a TrueFiSP at the MR-Linac for treatment planning; b contrast-enhanced computed tomography (CT) scan for treatment planning; c and d contrast-enhanced CT scan 3 months and 6 months after MR-guided radiotherapy of the liver metastasis

further metastases. A biopsy confirmed the diagnosis of a solitary pulmonary metastasis of the initial large-cell neuroendocrine carcinoma. Due to her age and severe pulmonary comorbidities, stereotactic body radiotherapy (SBRT) was recommended as treatment by an interdisciplinary tumor board. The patient was treated at the MRIdian Linac applying gated dose delivery with $8 \times 7.5 \mathrm{~Gy}$ prescribed to the $80 \%$ isodose, as the tumor was located adjacent to the thoracic wall (see - Fig. 2a, b). She tolerated the treatment well with only mild toxicity. The CT scans acquired during follow-up (3 and 6 months later) showed excellent treatment response with no signs of pneumonitis and only rare ground-glass opacity (- Fig. 2c, d).

The second patient (female, age 50) was diagnosed with locally advanced breast cancer in 2006 and was treated with neoadjuvant chemotherapy, breastconserving surgery, and adjuvant wholebreast radiotherapy. In 2017, pulmonary, hepatic, and bone metastases were detected and systemic treatment was initiated with fulvestrant/goserelin and palbociclib. In April 2019 progression limited to liver segments II/IV $(2.4 \mathrm{~cm} \times 5.6 \mathrm{~cm})$ was diagnosed, and the patient received gated MR-guided radiotherapy with $3 \times 15 \mathrm{~Gy}$ prescribed to the $65 \%$ isodose (- Fig. 3a, b). Palbociclib was paused during SBRT. Hepatic SBRT was well tolerated, the patient only suffered from mild nausea and fatigue. Follow-up MRI after 3 and 9 months showed treatment response with a reduction in size and perfusion of the irradiated hepatic metastasis (• Fig. 3c, d).

The third patient was diagnosed with a malignant melanoma of the conjunctiva of the left eye in 2014, which had been locally resected, preserving the eye ball. There were multiple recurrences over subsequent years that were treated by resection and systemic therapy with interferon. Due to further relapses, the orbit was exenterated in 2017 , followed by adjuvant radiotherapy. In 2018, multiple metastases in the lungs and the liver were diagnosed and treated with nivolumab/ipilimumab. The patient responded well to immunotherapy, but developed progression limited to the left adrenal gland. Therefore, MR-guided SBRT of the left adrenal metastasis was performed with $5 \times 10 \mathrm{~Gy}$ prescribed to the $80 \%$ isodose (- Fig. 4 a, b). The patient only suffered from mild dyspepsia during treatment. Follow-up CT scans illustrated excellent treatment response with complete remission of the irradiated adrenal gland metastasis after 3 and 6 months (- Fig. 4c, d).

The radiotherapy treatment at the MRLinac was well-tolerated by all patients. Patient acceptance was evaluated through patient-reported outcome questionnaires and revealed a positive or at least tolerable rating of the overall treatment [18].

\section{Plan comparison}

The treatment plan parameters of the MR-Linac and ITV-based plans are shown in - Table 1. Before a treatment plan is calculated by a physicist, the treating physician defines the clinical objectives for the respective plan, which consist of the dose prescription for the target volume (e.g., the tumor) and the dose constraints for the surrounding 


\section{Original article}

Table 1 Comparison of treatment plan parameters of the MR-Linac plans and the ITVapproach

\section{Tumor site}

Beams (MRL)

Beams (ITV)

Lung

Liver

Adrenal gland

CRT conformal radiotherapy, ITV internal target volume, $\boldsymbol{M R L}$ magnetic resonance-Linac

\begin{tabular}{|c|c|c|c|}
\hline Lung & MRL plan & ITV approach & Clinical goal \\
\hline PTV volume $\left[\mathrm{cm}^{3}\right]$ & 28.1 & 70.7 & - \\
\hline PTV V $60 \mathrm{~Gy}[\%]$ & 98.9 & 65.7 & $>95 \%$ \\
\hline Esophagus $\mathrm{D}_{0.5 \mathrm{~cm} 3}[\mathrm{~Gy}]$ & 19.0 & 6.8 & $<20$ Gy \\
\hline Heart $D_{0.5 \mathrm{~cm} 3}[\mathrm{~Gy}]$ & 2.0 & 11.8 & $<15 \mathrm{~Gy}$ \\
\hline Spinal cord $D_{0.1 \mathrm{~cm} 3}[\mathrm{~Gy}]$ & 8.8 & 4.7 & $<15$ Gy \\
\hline Central airways $D_{0.5 \mathrm{~cm} 3}[\mathrm{~Gy}]$ & 17.0 & 9.8 & $<20$ Gy \\
\hline Thoracic wall $\mathrm{D}_{30 \mathrm{~cm} 3}[\mathrm{~Gy}]$ & 26.6 & 42.4 & - \\
\hline Left lung $V_{20 G y}[\%]$ & 8.8 & 16.9 & - \\
\hline
\end{tabular}

Table 3 Dosimetric comparison of MRL plan and ITV approach for the liver

\begin{tabular}{|c|c|c|c|}
\hline Liver & MRL plan & ITV approach & Clinical goal \\
\hline PTV volume $\left[\mathrm{cm}^{3}\right]$ & 85.2 & 181.1 & - \\
\hline PTV V ${ }_{50 G y}[\%]$ & 99.1 & 98.0 & $>95 \%$ \\
\hline Bowel $D_{0.1 \mathrm{~cm} 3}[\mathrm{~Gy}]$ & 1.6 & 2.8 & $<21$ \\
\hline Spinal cord $D_{0.1 \mathrm{~cm} 3}[\mathrm{~Gy}]$ & 7.9 & 8.9 & $<18$ \\
\hline Stomach $D_{0.1 \mathrm{~cm} 3}[\mathrm{~Gy}]$ & 16.6 & 12.6 & $<21$ \\
\hline Liver GTV V $19 \mathrm{~Gy}\left[\mathrm{~cm}^{3}\right]$ & 274.3 & 266.5 & $<498.1$ \\
\hline \multicolumn{4}{|c|}{ 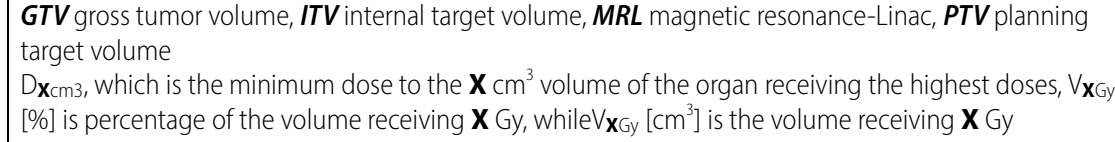 } \\
\hline
\end{tabular}

Table 4 Dosimetric comparison of MRL plan and ITV approach for the adrenal

\begin{tabular}{|c|c|c|c|}
\hline Adrenal & MRL plan & ITV approach & Clinical goal \\
\hline PTV volume $\left[\mathrm{cm}^{3}\right]$ & 41.7 & 145.9 & - \\
\hline PTV V V0Gy $[\%]$ & 97.6 & 88.8 & $>95 \%$ \\
\hline Bowel $D_{3 \mathrm{~cm} 3}[\mathrm{~Gy}]$ & 22.2 & 24.0 & $<24.50$ \\
\hline Kidney left mean dose [Gy] & 3 & 6.7 & $<10$ \\
\hline Kidney left $D_{33 \%}[\mathrm{~Gy}]$ & 1.6 & 3.1 & $<15$ \\
\hline Spinal cord $D_{0.1 \mathrm{~cm} 3}[\mathrm{~Gy}]$ & 8.5 & 16.0 & $<18$ \\
\hline Stomach $D_{3 \mathrm{~cm} 3}[\mathrm{~Gy}]$ & 18.9 & 24.50 & $<24.5$ \\
\hline
\end{tabular}

radiosensitive OARs. For SBRT, different groups have published certain dose constrains [22-24]. The dosimetry parameters for each plan, for target (PTV) coverage and normal tissue doses, are shown in $\bullet$ Tables 2, 3 and 4; the planning objectives against which these dosimetric parameters are judged are also shown.
Most of the objectives can be rejected if absolutely necessary, although dose to the spinal cord is considered a hard constraint. While the gated MR-Linac treatment plans fulfilled all clinical goals, only one ITV plan met all criteria.

As an ITV encompassing tumor motion during breathing had to be generated for the ITV plans, target volumes (PTV) were 2-3.5 times larger in the ITV plans compared with the MR-Linac plans (see - Tables 1, 2 and 3). Therefore, doses to the adjacent OARs were generally higher in the ITV plans than in the MR-Linac plans.

\section{Lung SBRT case}

For the lung ITV plan, only $66 \%$ of the PTV would have been encompassed by the $60 \mathrm{~Gy}$ isodose line, instead of the prescribed 95\% (see • Table 2; • Fig. 5). Moreover, the dose to the heart, thoracic wall, and left lung would have been considerably larger and closer to the prescribed clinical dosimetric constraints compared to the MR-Linac plans, whereas the MR-Linac plan approached the constraints for the maximum dose to esophagus and central respiratory tract.

\section{Liver SBRT case}

For the liver case, both plans fulfilled all the clinical objectives, with comparable dose-volume parameter values (see - Table 3; - Fig. 6). The dose distribution of both treatment plans is depicted in 0 Fig. 6.

\section{Adrenal SBRT case}

For the adrenal gland tumor, the PTV of the ITV-based plan overlapped in parts with the stomach, which resulted in a decreased dose coverage of the PTV compared with the MR-Linac plan, in order to meet the stomach dose constraint (see - Table 4; - Fig. 7). Hence, only $89 \%$ of the PTV was encompassed by the 50-Gy isodose. Although all other dose constraints were met in both plans, the dose to the spinal cord was substantially lower in the MR-Linac plan.

\section{Discussion}

Magnetic resonance-guided gated stereotactic radiotherapy allows for the application of favorable dosimetric treatment plans for moving tumors by decreasing irradiated volumes, with promising clinical results. It is assumed to be especially beneficial in abdominal or thoracic tumor sites [25-27]. For conventional ITVbased treatment plans, irradiated target volumes are considerably larger and may 

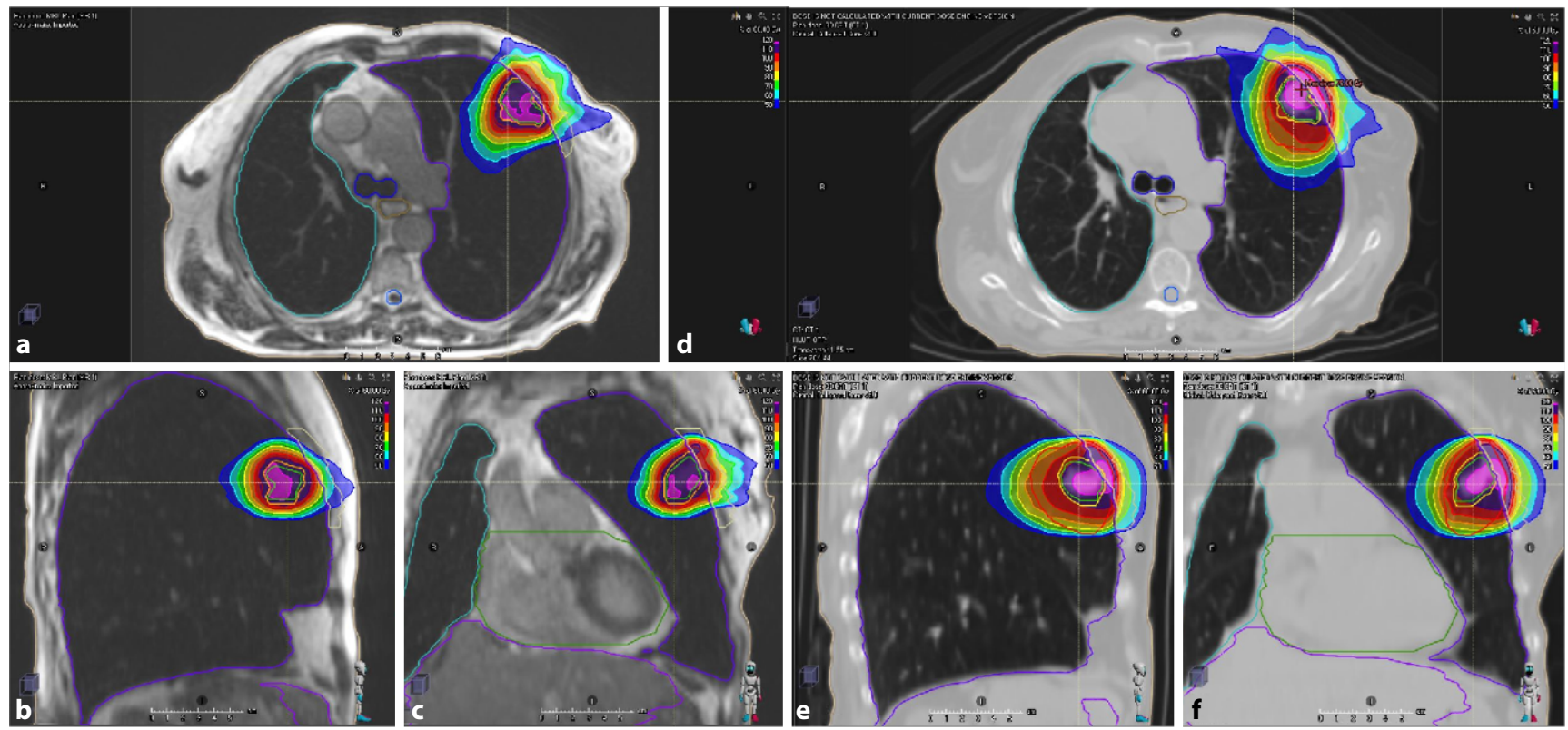

Fig. $5 \Delta$ Treatment plan for patient 1 (lung metastasis) as applied at the magnetic resonance(MR)-Linac using gated treatment in breath-hold shown as axial, sagittal and coronal images (a-c). Retrospectively generated computed tomography (CT)-based plan with internal target volume (ITV) in axial, sagittal and coronal images showing a larger treatment volume compared with the MR-Linac plan (d-f)
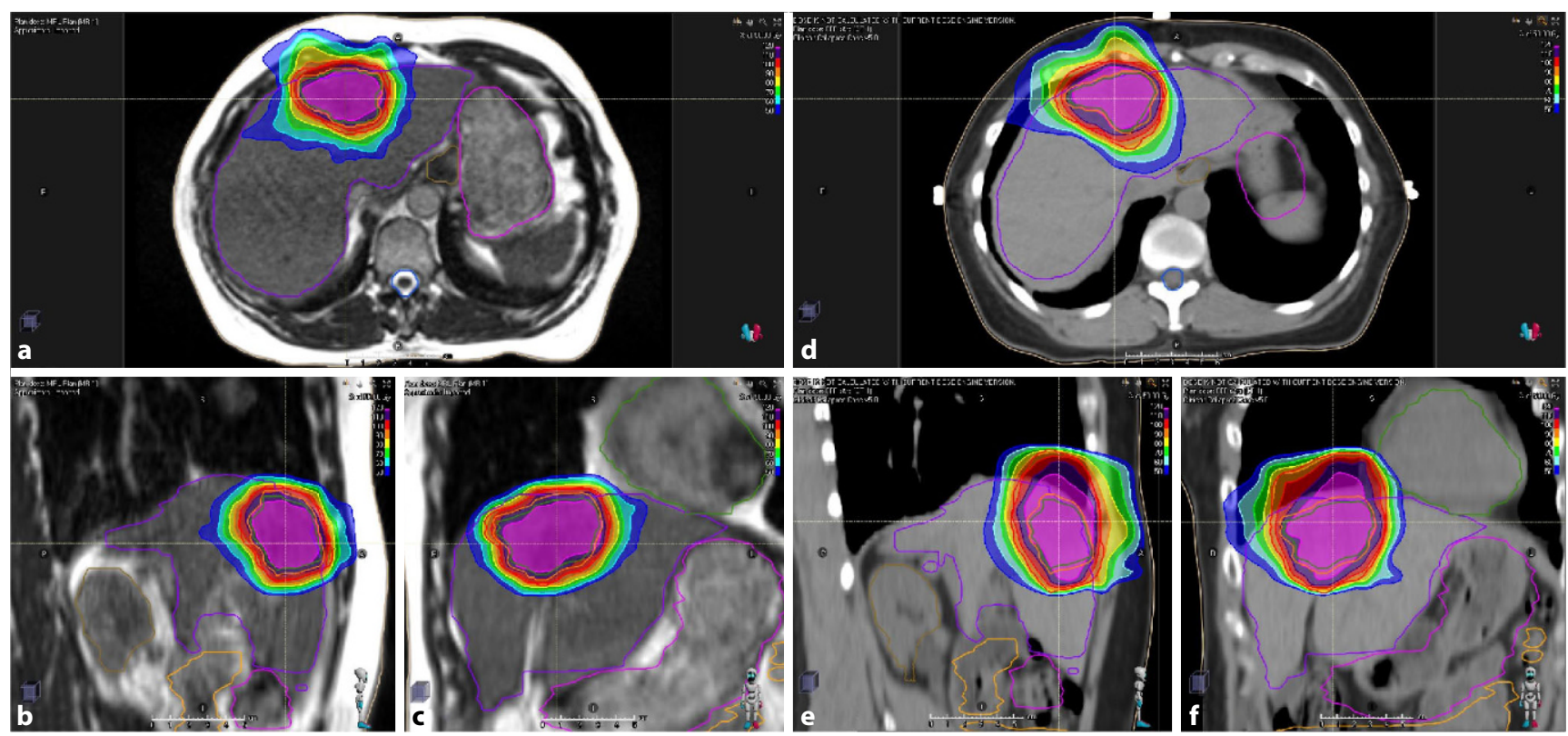

Fig. $6 \Delta$ Treatment plan for patient 2 (liver metastasis) as applied at the magnetic resonance(MR)-Linac using gated treatment in breath-hold (a-c: axial, sagittal and coronal images). Retrospectively generated computed tomography-based plan with internal target volume (ITV) showing a much larger treatment volume compared with the MR-Linac plan (d-f:axial, sagittal and coronal images)

overlap with critical organs, which can either lead to inferior target coverage or higher doses to OARs. For two of the three patients presented here, either less dose coverage of the PTV would have been achieved, or clinically a lower dose prescription would have been chosen if these patients would have been treated with an ITV approach. It should be noted that the ITV margins in this study were derived from the 2D cine-MRI (anterior-posterior and superior-inferior margin) as well as from the literature (lateral margin). Conventionally, they are ob- tained via $4 \mathrm{D}$ CT over several breathing cycles. However, the margins deduced by cine-MRI might be more realistic, since the whole treatment was evaluated instead of a limited number of breathing cycles on 1 day $[28,29]$. The lateral movement could not be evaluated on the 

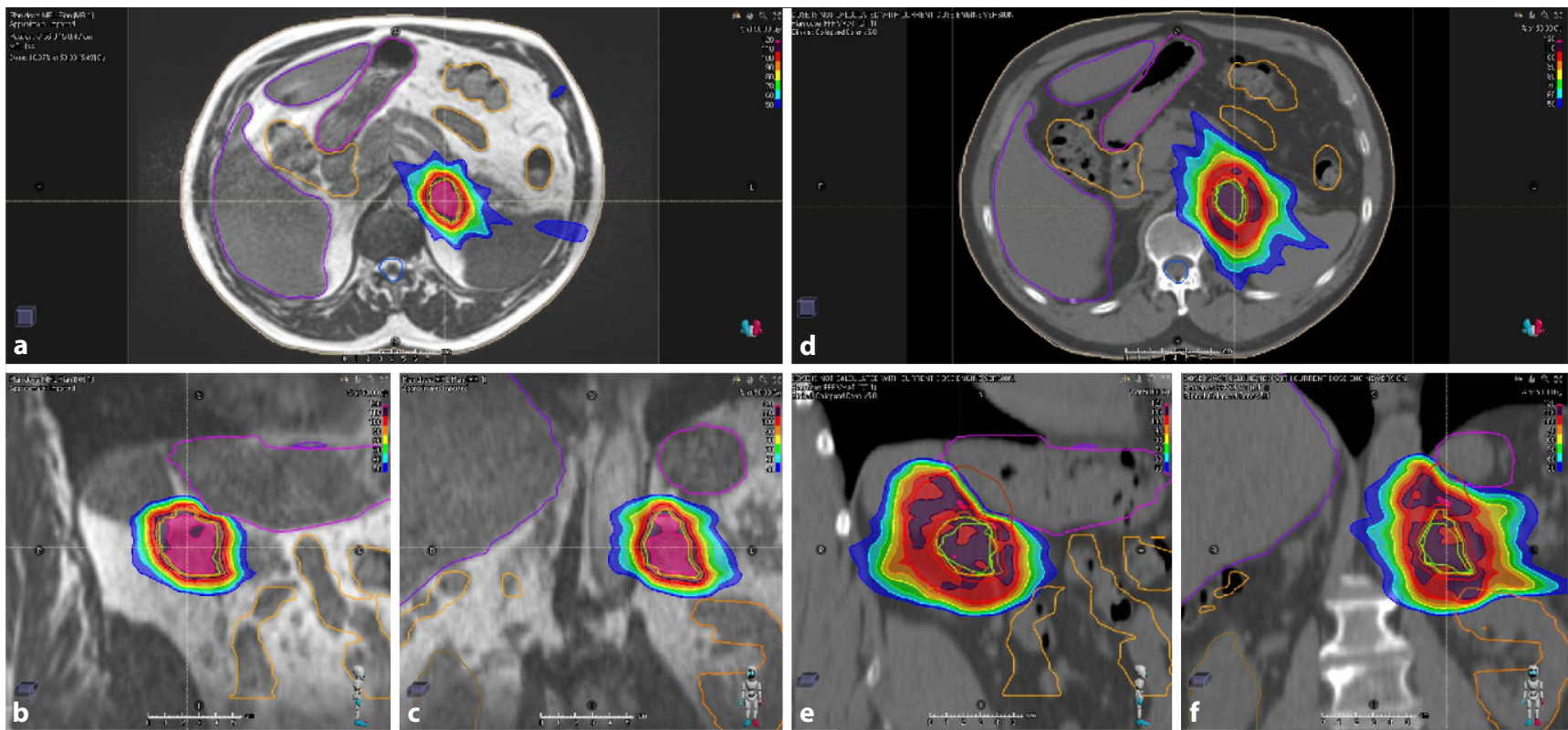

Fig. $7 \Delta$ Treatment plan for patient 3 (adrenal gland metastasis) as applied at the magnetic resonance(MR)-Linac using gated treatment in breath-hold (a-c: axial, sagittal and coronal images). Retrospectively generated computed tomography-based plan with internal target volume (ITV) showing a larger treatment volume compared with the MR-Linac plan (d-f: axial, sagittal and coronal images). In this case, the ITV extends into the right lung in order to geometrically cover the entire area of tumor location during breathing motion

sagittal cine-MRI data. For most patients and tumor sites, lateral motion is very limited, however.

In contrast to other techniques for respiratory beam gating, using radio-frequency transponders or $\mathrm{kV}$-gated treatment with fiducial markers, MR-guided gating is less invasive and is not accompanied by additional imaging radiation dose. Compared with surface-guided radiotherapy (SGRT), the MR-gated treatment offers increased accuracy, since the irradiated target is directly monitored and no assumption of a correlation between surface and target movement is required. Moreover, additional immobilization devices for abdominal compression, which can be used to minimize motion for ITV-based treatments but at the same time decrease patient comfort, can be omitted.

However, compared with the ITV approach, MR-guided treatments are more time-consuming. To facilitate reproducible breath-holds and thus shorten treatment time, many hospitals, including Heidelberg University Hospital, have installed a video monitor in the treatment room that shows the real-time cine-MR image to the patients, so that they can control the breath-hold by themselves $[18,30,31]$. With this approach, the patients have an active role during the duration of treatment, which is highly appreciated by the majority of patients [18]. In general, for most of the patients a dedicated breathing training is helpful in order to enable an efficient gating duty cycle. Due to the increased treatment time of the gated MR-Linac treatments, the patients have to lie still for at least $30 \mathrm{~min}$ in the treatment position. This has to be kept in mind when screening patients for the MR-Linac.

In order to further improve target tracking on the cine-MRI, dedicated contrast agents might be used. Especially for some liver tumors, the GTV cannot be sufficiently discriminated from the surrounding healthy liver in the native TRUFI sequence. Here, contrast agents such as gadoxetate disodium may help to visualize liver tumors [32]. Optimal contrasting of the hepatic lesion is usually detected after 15-20 min and usually persists during gated breath-hold SBRT [32].

The current standard MRI sequence for $3 \mathrm{D}$ as well as $2 \mathrm{D}$ cine-MR images on the MRIdian Linac is a TRUFI sequence, enabling straightforward delineation of target volumes and OARs. For abdominal and thoracic tumors, short imaging sequences are required to enable imaging in breath-hold without breathing artifacts. Thus, important issues are fast and robust image acquisition as well as geometric fidelity rather than diagnostic image quality. Until recently, only one sequence, namely, the TrueFISP sequence, was available and had to be used for target and OAR delineation at the MRIdian Linac. Together with a recent upgrade, a standard T1- and T2- as well as diffusion-weighted imaging (DWI) sequences are now also available at the MRIdian Linac. However, primary contouring is still only possible on the TrueFISP sequences. Furthermore, rigid as well as deformable registration of diagnostic contrast-enhanced MRI sequences is only achievable on a limited level.

Nevertheless, functional imaging like DWI for biological tumor response monitoring and prediction can be applied during the course of MR-guided treatments $[33,34]$. Shaverdian et al. showed that DWI is feasible on a low-field 0.35-T MRLinac in order to identify the individual response as well as potential treatment- 
resistant regions after chemoradiotherapy of rectal cancer [35]. Functional MRI may have potential use for biological dose adaption and dose escalation in the future [36], but further studies, especially on optimal imaging and adaption time points, are still required. Furthermore, MRI may also provide quantitative information about the actual delivered dose and its impact on the tumor as well as on normal tissue, which would allow for dose-compensating strategies and radiobiological modeling of tumor and normal tissue [34].

As MR-guided adaptive radiotherapy is highly personnel-intensive and timeconsuming, well-designed clinical trials are needed to identify the patients who benefit most from adaptive MR-guided radiotherapy. We recently published initial treatment experiences reporting a mean treatment time of $40 \mathrm{~min}$ for gated breath-hold SBRT, which is much longer compared with standard SBRT treatments of $10-15 \mathrm{~min}$ [18]. However, head-to-head comparative studies of CT-guided and MR-guided adaptive radiotherapy applying standard dose and fractionation might not be sufficient to demonstrate the true potential of MRguided adaptive radiotherapy, since MRguided adaptive radiotherapy allows for high-dose radiotherapy under circumstances in which treatment would not have been possible with conventional techniques [37].

The ultimate potential of the new MR-guided hybrid devices is immediate online adaptive treatment based on the anatomy of the day $[13,38]$. Radiation oncologists are now obliged to re-think the paradigms of total dose determination at the beginning of treatment and equal dose delivery during each single fraction. MR-guided adaptive radiotherapy holds promise in terms of applying the highest tolerated doses every single day depending on the current position of the tumor and the surrounding OARs. If dose escalation is not pursued, superior protection of critical structures from dose spillage is an alternative goal for plan adaptation. Initial studies have already reported mainly dosimetric advantages for online adaptation of MRguided stereotactic radiotherapy of pan- creatic, adrenal, or ultracentral thoracic malignancies among others $[11,13$, 39]. However, adaptive treatments further prolong treatment time to at least 45-70 min [30, 40]. Additional results of ongoing clinical studies are eagerly anticipated.

MR-guided radiation therapy enables online treatment plan adaption, options for real-time MR-based automated beam gating, and ultimately daily individualized radiotherapy. A close collaboration between radio-oncology and radiology as well as medical physics is needed to utilize the full potential in order to further improve tumor control and reduce side effects in cancer therapy.

\section{Conclusion}

With magnetic resonance (MR)-guided radiotherapy and cine-MR-based beam gating, moving tumors can be treated using smaller irradiated target volumes compared with conventional non-gated techniques. This results in dosimetrically beneficial treatment plans and promising clinical outcomes. Future studies will reveal the true potential of MRI for online biologically adapted radiation therapy and identify which patients will benefit most from this technique.

\section{Corresponding address}

PD Dr. med. Juliane Hörner-Rieber

Department of Radiation Oncology, Heidelberg University Hospital

Im Neuenheimer Feld 400, 69120 Heidelberg, Germany

juliane.hoerner-rieber@med.uni-heidelberg.de

\section{Compliance with ethical guidelines}

Conflict of interest. J. Hörner-Rieber and S. Klüter received speaker fees and travel reimbursement from ViewRay Inc. J. Hörner-Rieber received travel reimbursement from IntraOP Medical and Elekta Instrument $A B$. She also received a grant from IntraOP Medical. J. Debus received grants from CRI (The Clinical Research Institute GmbH), View Ray Inc., Accuray International, Accuray Incorporated, RaySearch Laboratories AB, Vision RT limited, Astellas Pharma GmbH, Merck Serono GmbH, AstraZeneca GmbH, Solution Akademie GmbH, Ergomed PLC Surrey Research Park, Siemens Healthcare $\mathrm{GmbH}$, Quintiles $\mathrm{GmbH}$, Phar- maceutical Research Associates $\mathrm{GmbH}$, Boehringer Ingelheim Pharma GmbH Co, PTW-Freiburg Dr. Pychlau $\mathrm{GmbH}$, Nanobiotix A.A., as well as IntraOP Medical. He has a research agreement with ViewRay Inc. and is a member of the Scientific Advisory Board of ViewRay Inc. (Oakwood, OH, USA). C.K.Spindeldreier, P.Hoegen, C. Buchele, C. Rippke, and E. Tonndorf-Martini declare that they have no competing interests.

For this article no studies with human participants or animals were performed by any of the authors. All studies performed were in accordance with the ethical standards indicated in each case.

The supplement containing this article is not sponsored by industry.

\section{References}

1. Bostel Tet al (2014) MR-guidance-a clinical study to evaluate a shuttle-based MR-linac connection to provide MR-guided radiotherapy. Radiat Oncol 9(1):12

2. Jaffray DA et al (2014) A facility for magnetic resonance-guided radiation therapy. Semin Radiat Oncol 24(3):193-195

3. Karlsson M et al (2009) Dedicated magnetic resonance imaging in the radiotherapy clinic. Int J Radiat Oncol Biol Phys 74(2):644-651

4. Henke LE et al (2018) Magnetic Resonance ImageGuided Radiotherapy (MRIgRT): A 4.5-year clinical experience. Clin Oncol 30(11):720-727

5. Fischer-ValuckBWetal (2017) Two-and-a-half-year clinical experience with the world's first magnetic resonance image guided radiation therapy system. Adv Radiat Oncol 2(3):485-493

6. Klüter S (2019) Technical design and concept of a 0.35T MR-Linac. Clin Translat Radiat Oncol 18:98-101

7. Raaymakers BW et al (2017) First patients treated with a $1.5 \mathrm{~T}$ MRI-Linac: clinical proof of concept of a high-precision, high-field MRI guided radiotherapy treatment. Phys Med Biol 62(23):L41-L50

8. Raaymakers BW et al (2009) Integrating a 1.5 T MRI scanner with a $6 \mathrm{MV}$ accelerator: proof of concept. Phys Med Biol 54(12):N229-N237

9. Green OL et al (2018) First clinical implementation of real-time, real anatomy tracking and radiation beam control. Med Phys 45:3728-3740

10. Burnet NG et al (2018) Zielvolumenkonzepte in der Strahlentherapie und ihre Bedeutung für die Bildgebung. Radiologe 58(8):708-721

11. Bohoudi $O$ et al (2019) Identification of patients with locally advanced pancreatic cancer benefitting from plan adaptation in MR-guided radiation therapy. Radiother Oncol 132:16-22

12. Acharya $S$ et al (2016) Magnetic resonance image guided radiation therapy for external beam accelerated partial-breast irradiation: evaluation ofdelivered doseand Intrafractional cavity motion Int J Radiat Oncol Biol Phys 96(4):785-792

13. Palacios MA et al (2018) Role of daily plan adaptation in MR-guided stereotactic ablative radiation therapy for adrenal metastases. Int $J$ Radiat Oncol Biol Phys 102(2):426-433

14. Rosenberg SA et al (2019) A multi-institutional experience of MR-guided liver stereotactic body radiation therapy. Adv Radiat Oncol 4(1):142-149

15. Bruynzeel AME et al (2019) A prospective singlearm phase 2 study of stereotactic magnetic resonance guided adaptive radiation therapy for 
prostate cancer: early toxicity results. Int J Radiat Oncol Biol Phys 105(5):1086-1094

16. Rudra S et al (2019) Using adaptive magnetic resonance image-guided radiation therapy for treatment of inoperable pancreatic cancer. Cancer Med 8(5):2123-2132

17. Hof $\mathrm{H}$ et al (2009) 4D-CT-based target volume definition in stereotactic radiotherapy of lung tumours: comparison with a conventional technique using individual margins. Radiother Oncol 93(3):419-423

18. Klüter $S$ et al (2020) First prospective clinical evaluation of feasibility and patient acceptance of magnetic resonance-guided radiotherapy in Germany. Strahlenther Onkol 196:691-698

19. Cusumano D et al (2018) Predicting tumour motion during the whole radiotherapy treatment: a systematic approach for thoracic and abdominal lesions based on real time MR. Radiother Oncol 129(3):456-462

20. Seppenwoolde $Y$ et al (2002) Precise and realtime measurement of 3D tumor motion in lung due to breathing and heartbeat, measured during radiotherapy. Int J Radiat Oncol Biol Phys 53(4):822-834

21. Dhont J et al (2018) The long- and short-term variability of breathing induced tumor motion in lung and liver over the course of a radiotherapy treatment. Radiother Oncol 126(2):339-346

22. Hanna GG et al (2018) UK consensus on normal tissue dose constraints for stereotactic radiotherapy. Clin Oncol (RColl Radiol) 30(1):5-14

23. Sterzing F et al (2014) Stereotactic body radiotherapy for liver tumors: principles and practical guidelines of the DEGRO Working Group on Stereotactic Radiotherapy. Strahlenther Onkol 190(10):872-881

24. Guckenberger M et al (2014) Definition of stereotactic body radiotherapy: principles and practice for the treatment of stage I non-small cell lung cancer. Strahlenther Onkol 190(1):26-33

25. Mittauer $\mathrm{K}$ et al (2018) A new era of image guidance with magnetic resonance-guided radiation therapy for abdominal and thoracic malignancies. Cureus 10(4):e2422

26. Corradini $S$ et al (2019) MR-guidance in clinical reality: current treatment challenges and future perspectives. Radiat Oncol 14(1):92

27. Bayouth JE, Low DA, Zaidi H (2019) MRI-linac systems will replace conventional IGRT systems within 15 years. Med Phys 46(9):3753-3756

28. Akino Y et al (2014) Evaluation of potential internal target volume of liver tumors using cine-MRI. Med Phys 41(11):111704

29. Cusumano Detal (2020) Reliability of ITVapproach to varying treatment fraction time: a retrospective analysis based on 2D cine MR images. Radiat Oncol 15(1):152

30. Tetar S et al (2018) Patient-reported outcome measurements on the tolerance of magnetic resonance imaging-guided radiation therapy. Cureus 10(2):e2236

31. Sahin B et al (2019) First 500 fractions delivered with a magnetic resonance-guided radiotherapy system: initial experience. Cureus 11(12):e6457

32. Wojcieszynski AP et al (2016) Gadoxetate for direct tumor therapy and tracking with real-time MRIguided stereotactic body radiation therapy of the liver. Radiother Oncol 118(2):416-418

33. Yang $Y$ et al (2016) Longitudinal diffusion MRI for treatment response assessment: preliminary experience using an MRI-guided tri-cobalt 60 radiotherapy system. Med Phys 43(3):1369-1373
34. White IM et al (2019) Realizing the potential of magnetic resonance image guided radiotherapy in gynaecological and rectal cancer. Br J Radiol 92(1098):20180670

35. Shaverdian N et al (2017) Feasibility evaluation of diffusion-weighted imaging using an integrated MRI-radiotherapy system for response assessment to neoadjuvant therapy in rectal cancer. Br J Radiol 90(1071):20160739

36. Hall WA et al (2019) The transformation of radiation oncology using real-time magnetic resonance guidance: a review. Eur J Cancer 122:42-52

37. Hunt A et al (2018) Adaptive radiotherapy enabled by MRI guidance. Clin Oncol 30(11):711-719

38. Werensteijn-Honingh AM et al (2019) Feasibility of stereotactic radiotherapy using a 1.5T MR-linac multi-fraction treatment of pelvic lymph node oligometastases. Radiother Oncol 134:50-54

39. Henke LE et al (2019) Stereotactic MR-guided online Adaptive Radiation Therapy (SMART) for ultracentral thorax malignancies: results of a phase 1 trial. Adv Radiat Oncol 4(1):201-209

40. van Timmeren JE et al (2020) Treatment plan quality during online adaptive re-planning. Radiat Oncol 15(1):203 